\title{
CENSO DAS PESSOAS COM DEFICIÊNCIAS E AS SUAS FUNCIONALIDADES NA CIDADE DE MARACAÍ/SP
}

\author{
Silas de Oliveira Damasceno ${ }^{1}$, José Henrique Piedade Cardoso ${ }^{2}$, Natália Trindade da Silva ${ }^{3}$ \\ ${ }^{1}$ Universidade Estadual Paulista "Júlio Mesquita Filho" - UNESP, Residência em Fisioterapia Aplicada à Neurologia, \\ Presidente Prudente/SP. E-mail: silas.damasceno10@hotmail.com. \\ 2Universidade Estadual Paulista “Júlio Mesquita Filho" - UNESP, Residência em Geriatria e Gerontologia , Presidente \\ Prudente/SP \\ ${ }^{3}$ Universidade Paulista - UNIP, Curso de Fisioterapia, Assis, SP. E-mail: nataliatrindades@gmail.com
}

\section{RESUMO}

A deficiência é um assunto complexo e que necessita de uma melhor abordagem pelos fisioterapeutas. O objetivo foi levantar o número de pessoas com deficiências (PCD), os tipos de deficiências e a funcionalidade desta população na cidade de Maracaí/SP. O levantamento dos dados do censo foi realizado através de um questionário previamente testado e a funcionalidade avaliada por meio das escalas de Barthel e equilíbrio de Tinetti. Foram percorridos pelo Censo $66,64 \%$ dos bairros e foram localizadas 42 pessoas com deficiência, sendo que $77 \%$ ( $n=32$ ) participaram efetivamente da pesquisa. Os resultados mostraram deficiência intelectual $(46,87 \%)$, física $(28,12 \%)$, múltipla $(12,5 \%)$, auditiva $(6,25 \%)$ e visual $(6,25 \%)$. Neste estudo evidenciou os diferentes tipos de deficiências, tendo a deficiência intelectual como maior acometimento e os participantes apresentaram alto risco de quedas e comprometimento moderado na funcionalidade.

Palavras-chave: Deficiência, censo, inclusão social, fisioterapia.

\section{CENSUS OF THE PEOPLE WITH DISABILITIES AND THEIR FUNCTIONALITIES IN THE CITY OF MARACAÍ/SP}

\begin{abstract}
Disability is a complex subject and requires a better approach by the physiotherapists. The objective was to raise the number of people with disabilities (PWD), types of disabilities, and functionality of this population in the city of Maracaí/SP. The study of census data was carried out through a previously tested questionnaire, and functionality evaluated through Barthel scales and Tinetti balance. We ran with the questionnaire in $66.64 \%$ of the neighborhoods, where we located 42 people with disabilities, and $77 \%(n=32)$ that participated effectively in the survey. The results showed intellectual disability $(46.87 \%)$, physical $(28.12 \%)$, multiple $(12.5 \%)$, auditory $(6.25 \%)$ and visual (6.25\%). This study showed the differents types of disabilities being the intellectual disability the most affected and the participants they are in the adaptive phase featured at high risk of falls and moderate commitment in functional capacity.
\end{abstract}

Keywords: Disability, census, social inclusion, physiotherapy. 


\section{INTRODUÇÃO}

A temática que abrange a deficiência é um assunto que advém desde outrora, muito complexa e que necessita de uma abordagem que resulte em uma resposta mais simples no que tange a caracterização deste grupo populacional, haja vista que, se deve criar uma nova perspectiva para um problema que circunda a sociedade e suas implicações na coletividade. Tal pensamento vem adquirindo um engajamento populacional cada vez maior, uma vez que ao longo dos anos vem sofrendo mutações permeadas por períodos específicos da história da humanidade ${ }^{1-}$ 2-3.

Essa evolutiva concebeu a legislação atual, deixando as pessoas com deficiência em um patamar menos segregativo. De forma a assegurar os direitos igualitários, criou-se a Constituição Federal de 1988, na qual estabeleceu os principais fundamentos da República Federativa do Brasil e que foi ratificada pela Lei no 7.853 de outubro de 1989, onde percorre acerca dos benefícios totalitários para pessoas com deficiência ${ }^{4-5}$.

Entretanto, apesar da implantação desta lei constitucional, existe uma lacuna para efetivação dos direitos presentes nesta norma, tanto na vertente de uma maior divulgação de informações, assim como de uma mobilização da população para cobrar de forma efetiva dos governantes o que se consta no documento ${ }^{1}$.

Logo, compreender este contingente populacional e suas características é um processo fundamental para elaboração das metas, pois sua população não é imutável, assim como na cidade de Maracaí/SP que é parte fundamental deste estudo.

Maracaí é uma cidade localizada a $473 \mathrm{~km}$ da capital São Paulo e com uma área estimada em $543 \mathrm{~km}^{2}$, que consta com uma população, segundo o último levantamento com 13.344 habitantes, e uma equivalência substancial de 6.670 para homens e 6.674 para mulheres, dispersos tanto na área urbana como na área rural, sendo o último suplantado pelo primeiro, que contém 12.098 habitantes. De economia essencialmente agrícola apresenta um IDH (Índice de Desenvolvimento Humano) de 0,773, distribuída em dois distritos: Santa Cruz da Boa Vista e São José das Laranjeiras ${ }^{6-7}$.

Contudo, por se tratar de uma cidade em desenvolvimento e com crescente número populacional, ficou evidente a necessidade de um levantamento de pessoas com deficiências. Por inércia, o contingente populacional analisado, obteve seus direitos como ser humano sem afetar sua condição como cidadão, e a fisioterapia, como curso da área da saúde, tem papel primordial no levantamento dessas informações, uma vez que através deste trabalho elucidou caminhos para que objetivos terapêuticos e sociais fossem traçados.

Assim, o objetivo deste estudo foi localizar, contar e classificar os tipos de deficiências e as funcionalidades das Pessoas com Deficiências (PCD) na cidade de Maracaí/SP.

\section{MÉTODO}

O censo foi realizado através de um estudo de campo, epidemiológico e de caráter transversal. O levantamento dos dados ocorreu após a aprovação da Comissão de Ética e Pesquisa (CEP) da Universidade Paulista (UNIP), sob o parecer de número 1.103.788, CAAE 45323415.6.0000.5512. A investigação dos dados realizou-se por meio de um questionário previamente testado e escalas selecionadas. A participação do participante deu-se de maneira voluntária, por meio da assinatura prévia do Termo de Consentimento Livre e Esclarecido (TCLE).

Foram aceitos voluntários para coleta de dados; inicialmente foi aberto aos alunos dos cursos da área da saúde, como não houve voluntário, abriu-se para todo cidadão que se disponibilizou participar da pesquisa e que fosse capaz de cumprir o treinamento, entretanto, também não houve voluntários. Então, a busca foi realizada pelo pesquisador do projeto, que 
afirma, que com isso pôde se inteirar completamente com o estudo, e assim o recomenda para posteriores estudos que apresentarem a mesma metodologia.

Os materiais utilizados compreenderam: Um questionário validado e estruturado por especialistas na área, duas cadeiras rígidas e sem braços, uma folha em branco, uma caneta, um relógio, uma escada de dois degraus, um crachá de identificação do pesquisador, a) uma Escala de Funcionalidade de Barthel para AVD, b) uma Escala de Equilíbrio de Tinetti, primeira parte, e c) uma Escala Mini Exame de Estado Mental:

A) Escala de Equilíbrio de Funcionalidade de Barthel ${ }^{8,9,10}$ : avalia a independência funcional dos participantes, contida em atividades que variam de $0,5,10$ ou 15 pontos, tendo pontuação mínima de 0 e máxima de 100; menor independência e maior independência, respectivamente.

B) Escala de Equilíbrio de Tinetti ${ }^{11,12,13}$ : avalia o equilíbrio do participante. Apresenta 9 itens que são pontuados de 0 a 2 pontos, totalizando 16 pontos, sendo o escore mais alto o melhor nível de equilíbrio e o menor, inversamente proporcional.

C) Escala Mini Exame de Estado Mental ${ }^{14,15,16}$ : é uma escala que faz a avaliação da cognição do participante. Possui 5 itens, podendo o participante, atingir até 5 pontos na orientação espacial, na orientação temporal e na atenção e cálculo; atingir no máximo 3 pontos na memória imediata e na memória de evocação e 9 pontos na linguagem, totalizando 30 pontos de pontuação máxima.

Foram participantes desta pesquisa todas as PCD moradoras da área urbana da cidade de Maracaí/SP. A busca dos dados consistiu percorrer os bairros da cidade, informando sobre os tipos de deficiências de modo geral e questionando de casa em casa, se existia participante que queria aderir à pesquisa, devendo, obrigatoriamente, possuir qualquer tipo de deficiência (física, visual, auditiva, intelectual, motora ou múltipla) e que aceitasse anuir ao TCLE.

O empregado das escalas foi desenvolvido no ambiente natural do participante, em local iluminado, ventilado, sem ruídos que atrapalhassem o entendimento das perguntas e comandos e sem desníveis no chão. Durante a aplicação das escalas e questionário, para o participante não ser influenciado nas respostas, apenas estavam presentes no ambiente: $O$ aplicador, o participante e um responsável para auxiliar nas respostas do participante que não conseguia verbalizar, segundo relato inicial do responsável.

Inicialmente, foi aplicado o questionário para conhecer os dados pessoais, condições socioeconômicas e não cansar o participante antes do final da utilização das escalas. Em seguida foi aplicado a Escala de Mini Exame de Estado Mental, na qual o participante podia realizar na cadeira que já se encontrava, somente passando pela Escala de Equilíbrio de Tinetti, após a última escala citada ser concluída, e por fim, a Escala de Funcionalidade de Barthel, pois esta precisava realizar transferência e subir escadas, necessitando um dispêndio de energia maior dos participantes.

$\mathrm{Na}$ aplicação do questionário, o pesquisador utilizava falas simples e um tom claro para evitar dúvidas e em seguida anotava a resposta. E na aplicação das escalas, o comando de voz utilizado foi de falas positivas, que incentivasse o participante: "Você consegue!" ou "força, falta pouco!".

Os resultados foram tabulados no Microsoft Excel ${ }^{\circledR}$ e apresentados de forma descritiva, por meio de média e desvio padrão para dados nominais e em percentuais para os dados numéricos. 


\section{RESULTADOS}

O presente estudo foi desenvolvido na cidade de Maracaí estado de São Paulo, percorrendo $66,64 \%$ dos bairros e foram localizadas 42 pessoas com deficiências, sendo que $77 \%$ $(n=32)$ participaram efetivamente da pesquisa.

$\mathrm{Na}$ sua totalidade, $59,37 \%$ eram do sexo feminino e $40,62 \%$ do sexo masculino, tendo média de idade para o primeiro de 41,69 anos e para o segundo de 46,31 anos, respectivamente, e a média geral de idade foi de $44 \pm 23,80$. Segundo a naturalidade, $43,75 \%$ eram naturais da cidade em questão e $56,25 \%$ proveniente de outras regiões do Brasil.

Dentre as deficiências encontradas, a que apresentou maior percentual foi à deficiência intelectual $46,87 \%$, seguida da física $28,12 \%$, múltipla (12,5\%), auditiva $(6,25 \%)$ e visual $(6,25 \%)$.

Em relação ao estado civil dos participantes, as mulheres apresentaram serem em sua maioria solteiras $55,84 \%, 15,38 \%$ casadas, $7,69 \%$ amasiadas, $15,38 \%$ viúvas e $7,69 \%$ divorciadas. Não obstante, os homens também em sua plenitude, $47,36 \%$ eram solteiros, $42,10 \%$ casados, $5,26 \%$ viúvos, $5,26 \%$ divorciados e amasiados não foram encontrados.

No prisma da escolaridade $37,5 \%$ dos entrevistados não possuíam nível de escolaridade, entretanto, o antigo primário foi frequentado por $43,75 \%$, ensino médio por 3,12\%, 12,5\% antigo ginásio e 3,12\% se enquadraram em outros.

No enfoque financeiro, $87,5 \%$ não exerciam função remunerada contra $12,5 \%$ que realizavam alguma atividade assalariada. Disseram sim ao recebimento de auxílio financeiro $68,75 \%$ e não $31,25 \%$ dos participantes, sendo que, $72,72 \%$ viviam da aposentadoria, e destes, 93,75\% recebiam apenas um salário mínimo e 6,25\% mais de um salário mínimo, inferindo diretamente no nível de saúde do participante.

Quanto ao uso de transporte urbano $34,38 \%$ relataram o uso deste meio de transporte e $65,62 \%$ negaram. Foi questionado se o participante frequentava o comércio local, no qual, $65,62 \%$ afirmaram que sim e 34,38\% não, quase na mesma proporção, 56,25\% afirmaram serem participantes em bares, festas e afins e $43,75 \%$ negaram a presença nestes ambientes.

Na mesma vertente, a Escala Mini Exame de Estado Mental apresentou média de 13,94 pontos de 30 possíveis, a Escala de Equilíbrio de Tinetti 9,44 de uma totalidade de 16 pontos e a Escala de Funcionalidade de Barthel para AVD dos 100 pontos prováveis apresentou uma média de 64,34 pontos.

\section{DISCUSSÃO}

O presente estudo evidenciou que na cidade de Maracaí/SP na população analisada a deficiência intelectual é o comprometimento mais presente, assim como possuem uma tendência para a demência e risco de quedas que levam ao uma dificuldade moderada na funcionalidade.

O Instituto Brasileiro de Geografia e Estatística (IBGE) realizou pesquisas censitárias para inferir na composição populacional e dar um pressuposto para uma política mais igualitária, sendo no ano de 2010, a atualização dos dados colhidos no ano 2000, onde se obteve inúmeros dados, inclusive sobre pessoas com deficiências ${ }^{7-17}$.

Segundo os dados mais recentes, 45.606 .048 dos brasileiros, correspondendo a $23,9 \%$ da população total têm algum tipo de deficiência: auditiva, intelectual, visual ou motora, sendo as mulheres em maior acometimento do que os homens, $26,5 \%$ e $21,2 \%$, respectivamente, o que entra em consonância com o atual estudo em relação ao maior acometimento no sexo feminino ${ }^{7}$.

Ainda sobre a pesquisa relatada anteriormente, a deficiência visual apresentou maior índice com $18,6 \%$, deficiência motora com $7 \%$, deficiência auditiva $5,10 \%$ e deficiência intelectual $1,40 \%$ da população, ordem diferente dos dados apresentados no atual estudo ${ }^{7}$.

Os dados colhidos pelo IBGE refletem a perspectiva dos entrevistados quanto ao tipo de deficiência que os acometem, no qual se fizermos uma comparação, o Censo 2000, apresentava 24.600.256, ou 14,5\% dos brasileiros relatantes de terem pelo menos um dos tipos de deficiências, 
enquanto no Censo 2010, este número subiu para 45.606 .048 , ou $23,9 \%$, de forma a evidenciar que este tipo de levantamento se faz necessário para subsidiar as pessoas com deficiência em suas prerrogativas e baseados nos princípios dos direitos humanos ${ }^{17,7}$.

Corroborando com as pesquisas supracitadas, no centro-oeste paulista, uma pesquisa de cunho epidemiológico realizada na cidade de Assis-SP, com aproximadamente 95.156 habitantes, percorreu 64,5\% dos bairros, no qual evidenciou 905 pessoas com deficiências sendo $69,7 \%$ de participantes efetivos, equiparando com este estudo, que obteve $77 \%$ de participantes ativos, mostrando que esta população está ciente frente sua participação na construção social ${ }^{18}$.

Dentre as grandes regiões, a região Centro-Oeste, perímetro onde foi realizado este estudo, apresentou a segunda menor taxa de deficiente qualificado com pelo menos uma deficiência, compreendendo $22,51 \%$ e a região Sul do país alcançou a menor taxa com $22,5 \%$ da população analisada. O estado de São Paulo, região que se encontra este estudo, apresentou $22,6 \%$, tendo o segundo menor percentual do Brasil ${ }^{7}$.

Estes dados evidenciam que a fisioterapia representa importante papel na vida das PCD. Em algum momento da vida destas pessoas, a Fisioterapia age na promoção da saúde, reabilitação funcional e/ou aquisição de habilidades. Leis têm sido criadas na garantia de que estas pessoas tenham seus direitos garantidos junto à sociedade, assim, localizá-los e saber de suas habilidades é necessidade para estas reivindicações, que envolvem os setores da saúde, educação e lazer.

As limitações do estudo referem-se ao pequeno número da amostra, aos comprometimentos motores heterogêneos, patologias e idades diferentes e que somados, puderam influenciar nos resultados encontrados. Logo, sugere-se que estudos futuros, levem em consideração tais levantamentos para melhores resultados, uma vez que estes dados mostram a necessidade de novas pesquisas para delimitar o tema e dar subsídios para PCD e servir de fomento para que outros municípios realizem tal pesquisa.

\section{CONCLUSÃO}

Conclui-se que houve predominância da deficiência intelectual, com comprometimento neurológico moderado inferindo-se em um quadro de demência e com acometimento mais em adulto-jovens. Quanto ao aspecto de equilíbrio, a população encontra-se na fase adaptativa com alto risco para quedas e no espectro funcional os participantes identificados apresentaram um comprometimento moderado na funcionalidade.

\section{REFERÊNCIAS}

1. Sassaki RK. Inclusão: Construindo uma sociedade para todos. 7ạ Ed. Rio de janeiro: WVA, 2006.

2. Aranha MSF. Programa de Educação Inclusiva: Direito à Diversidade: A Fundamentação Filosófica. 2a Ed. Brasília: Ministério da Educação, Secretaria de Educação Especial, 2006.

3. lamamoto MV, Carvalho R. Relações Sociais e Serviço Social no Brasil: Esboço de uma interpretação histórico-mercadológica. $3^{\circ}$ ed. Cortez. São Paulo-SP: Outubro, 1981.

4. Brasil, Presidência da República Casa Civil Subchefia para Assuntos Jurídicos. Constituição da República Federativa do Brasil de 1988.

5. Brasil, Presidência da República Casa Civil Subchefia para Assuntos Jurídicos. Lei no 7.853, de 24 de outubro de 1989. 
6. Prefeitura Municipal de Maracaí. Sítio online, 2018.

7. Oliveira LMB, Secretaria de Direitos Humanos da Presidência da República (SDH/PR), Secretaria Nacional de Promoção dos Direitos da Pessoa com Deficiência (SNPD), Coordenação Geral do Sistema de Informações sobre a Pessoa com Deficiência. Cartilha do Censo 2010: Pessoas com Deficiência. Brasília: SDH-PR/SNPD, 2012.

8. Minosso JSM, Amendola F, Alvarenga MRM, Oliveira MAC. Validação, no Brasil, do Índice de Barthel em idosos atendidos em ambulatórios. Acta Paul Enferm. 2010; 23(2): 218-23. DOI: http://dx.doi.org/10.1590/S0103-21002010000200011.

9. Bortoli CG, Piovezan MR, Juliato Piovesan EJ, Zonta MB. Equilíbrio, quedas e funcionalidade em idosos com alteração da função cognitiva. Rev. Bras. Geriatr. Gerontol., Rio de Janeiro. 2015; 18(3): 587-597. DOI: http://dx.doi.org/10.1590/1809-9823.2015.14057.

10. Lobo AJS, Santos L, Gomes S. Nível de dependência e qualidade de vida da população idosa. Rev Bras Enferm. 2014; 67(6): 913-9. DOI: http://dx.doi.org/10.1590/0034-7167.2014670608.

11. Pires CM. Papel da Intervenção Psicomotora na Autonomia e Independência do Idoso Institucionalizado [dissertação de mestrado]. Vila Real: Programa de Pós-graduação em Gerontologia: Atividade Física e Saúde no idoso, pela Universidade de Trás-os-Montes e Alto Douro, 2014.

12. Pedro, EM, Amorim, DB. Análise comparativa da massa e força muscular e do equilíbrio entre indivíduos idosos praticantes e não praticantes de musculação. Conexões: revista da Faculdade de Educação Física da UNICAMP, Campinas, v. 6, ed. especial, p. 174-183, jul. 2008 ISSN: 1983 - 9030. DOI: https://doi.org/10.20396/conex.v6i0.8637823.

13. Sacco IDN, Bacarin TA, Watari R, Suda EY, Canettieri MG, Souza LC, et al. Envelhecimento, atividade física, massa corporal e arco plantar longitudinal influenciam no equilíbrio funcional de idosos?. Rev. bras. Educ. Fís. Esp. 2008; São Paulo, v.22, n.3, p.183-91. DOI: https://doi.org/10.1590/S1807-55092008000300002.

14. Chapman KR, Bing-Canar H, Alosco ML, Steinberg EG, Martin B, Chaisson C, et al. Mini Mental State Examination and Logical Memory scores for entry into Alzheimer's disease trials. Alzheimer's Research \& Therapy. 2016; 8(1): 9. DOI: https://doi.org/10.1186/s13195-016-0176-z

15. Votruba KL, Persad C, Giordani B. Cognitive Deficits in Healthy Elderly Population With "Normal" Scores on the Mini-Mental State Examination. Journal of Geriatric Psychiatry and Neurology. 2016; 29(3): 126-132. DOI: http://dx.doi.org/10.1177/0891988716629858.

16. Santana I, Duro D, Lemos R, Costa V, Pereira M, Simões MR, et al. Mini-Mental State Examination: Avaliação dos Novos Dados Normativos no Rastreio e Diagnóstico do Défice Cognitivo. Revista Científica da Ordem dos Médicos. Acta Med Port. 2016; 29(4): 240-248. DOI: http://dx.doi.org/10.20344/amp.6889.

17. Ministério do Planejamento, Orçamento e Gestão. Instituto Brasileiro de Geografia e Estatística - IBGE. Censo Demográfico 2000: Características Gerais da População. ISSN 0104-3145. Censo demogr. 2000; Rio de Janeiro, p. 1-178. 
18. Santos BM, Paes HHF, Junior PRR, Pacheco SCS, Pacheco CRS. Doenças, fármacos e terapêutica em idosos com deficiência. J Health Sci Inst. 2012; 30(2): 181-5. 